\title{
Transient receptor potential ankyrin 1 mediates cisplatin-induced apoptosis in renal tubular cells via calcium-dependent signaling pathway
}

\author{
Xianglong Meng ${ }^{1 \#}$, Yaling Zhang ${ }^{1 \#}$, Yuexian $\mathrm{Ma}^{2}$, Yaqin $\mathrm{Du}^{3}$, Cuo Gan ${ }^{3}$, Jing Feng ${ }^{4}$, Fei Deng ${ }^{1,3}$ \\ ${ }^{1}$ Department of Nephrology, Sichuan Academy of Medical Sciences \& Sichuan Provincial People's Hospital, University of Electronic Science and \\ Technology of China, Chengdu, China; ${ }^{2}$ Department of Nephrology, Affiliated Hospital of Chengdu University of Traditional Chinese Medicine, \\ Chengdu, China; ${ }^{3}$ Department of Nephrology, Jinniu Hospital of Sichuan Provincial People's Hospital \& Chengdu Jinniu District People's Hospital, \\ Chengdu, China; ${ }^{4}$ Department of Traditional Chinese Medicine, Sichuan Academy of Medical Sciences \& Sichuan Provincial People's Hospital, \\ University of Electronic Science and Technology of China, Chengdu, China \\ Contributions: (I) Conception and design: J Feng, F Deng, X Meng, Y Zhang; (II) Administrative support: C Gan; (III) Provision of study materials or \\ patients: Y Ma, Y Du; (IV) Collection and assembly of data: X Meng, Y Ma; (V) Data analysis and interpretation: X Meng, Y Zhang; (VI) Manuscript \\ writing: All authors; (VII) Final approval of manuscript: All authors. \\ \#These authors contributed equally to this work. \\ Correspondence to: Jing Feng. Department of Traditional Chinese Medicine, Sichuan Academy of Medical Sciences \& Sichuan Provincial People's \\ Hospital, 32\# W. Sec 2, 1st Ring Rd. (Qingyang Taoist Temple Diagonally Opposite), Chengdu 610072, China. Email: fengjing_here@126.com; \\ Fei Deng. Department of Nephrology, Sichuan Academy of Medical Sciences \& Sichuan Provincial People's Hospital, 32\# W. Sec 2, 1st Ring Rd. \\ (Qingyang Taoist Temple Diagonally Opposite), Chengdu 610072, China. Email: dengfei_here@163.com.
}

Backgroundk Cisplatin has been a vital drug used for tumor treatment because of its excellent effect on numerous malignant solid cancers. Nonetheless, its nephrotoxicity is non-negligible in clinical practice. This study aims to provide a new understanding of the molecular mechanism of transient receptor potential ankyrin 1 (TRPA1) in cisplatin-induced renal apoptosis.

Methods: We evaluated the effect on apoptosis, TRPA1 expression, and intracellular calcium concentration of human kidney 2 (HK-2) cells induced by diamminedichloroplatinum (DDP). Additionally, we also assessed DDP-induced apoptosis, the expression of Bax, caspase3, cleaved-cas3, p53, Bcl-2 and intracellular calcium concentration combined with HC-030031 and/or pifithrin- $\alpha$. The effect of FK506 on apoptosis of HK-2 cells induced by DDP and the expression of the nuclear factor of activated $\mathrm{T}$ cells (NFAT) protein treated with HC-030031, pifithrin- $\alpha$, and/or FK506 were also explored.

Results: The results showed that apoptosis, TRPA1 expression, and intracellular calcium concentration of HK-2 cell induced by DDP were enhanced in a dose-dependent manner. HC-030031 and pifithrin- $\alpha$ relieved apoptosis, and intracellular calcium concentration and the expression of NFAT and phospho-NFAT (p-NFAT) were induced by DDP. HC-030031 combined with pifithrin- $\alpha$ further aggravated the abovementioned tendency, including relieved apoptosis, intracellular calcium concentration, and NFAT and p-NFAT expression. HC-030031 and FK506 decelerated the apoptosis, and NFAT and p-NFAT expression of HK-2 cells was induced by DDP, while simultaneous treatment with HC-030031 and FK506 further decreased apoptosis and protein expression. However, the expression of Bcl-2 increased when HC-030031, pifithrin- $\alpha$, or FK506 was used alone, and HC-030031 combined with pifithrin- $\alpha$ or FK506 further improved the expression of Bcl-2.

Conclusions: TRPA1 mediates cisplatin-induced apoptosis in renal tubular cells via the calcineurinnuclear factor of activated T-cells-p53 signaling pathway.

Keywords: Cisplatin; nephrotoxicity; apoptosis; TRPA; calcineurin; NFAT2 
Submitted Jun 16, 2021. Accepted for publication Jul 28, 2021.

doi: 10.21037/apm-21-1867

View this article at: https://dx.doi.org/10.21037/apm-21-1867

\section{Introduction}

Due to its ability to inhibit cell division, cisplatin (cisdiamminedichloroplatinum, cis-DDP) has been one of the most indispensable drugs in the chemotherapeutic treatment of tumors (1-3). It is widely used in the treatment of testicular, ovarian, non-small cell lung, head, neck, and cervical carcinoma and many other types of cancers (2-5). However, its side-effects have also presented many challenges to clinicians. The known severe side-effects caused by cisplatin include: neurotoxicity, ototoxicity, renal toxicity, myelosuppression, nausea, and vomiting (4,6-9). Despite certain precautions used in clinical practice (10-12), nephrotoxicity caused by cisplatin remains the main barrier that limits the use and efficacy of cisplatin in cancer therapy. Currently, about 20-30\% patients develop nephrotoxicity when receiving cisplatin $(4,13)$. Importantly, the most common and serious clinical manifestation of nephrotoxicity is acute kidney injury (AKI) (14). Apoptosis (15) and inflammation (16) have been considered as the potential mechanisms of nephrotoxicity caused by cisplatin. The nephrotoxicity mainly occurs in the proximal tubule, and the accumulation of cisplatin in the proximal tubule epithelial cells can lead to cell apoptosis and necrosis $(17,18)$.

Transient receptor potential ankyrin 1 (TRPA1), a member of the transient receptor potential (TRP) ion channel family, is a ligand-gated non-selective cation channel (19). Except for the major function of thermosensation, TRPA1 has been proven to play a vital role in the transmission and integration of nociceptive signals owing to its predominant expression in nociceptive nerve fibers $(20,21)$. Additionally, more and more researchers support the view that TRPA1 also plays an important role in inflammation (20). Certainly, as a nonselective calcium-permeable channel, TRPA1 could be involved in calcium-regulated signaling pathways. Calcium is an essential second messenger, which connects membrane receptor activation and downstream signaling transduction, and plays a vital role in numerous cell physiological processes such as cell excitability, apoptosis, vitality, and transcription (22). The activation of TRPA1 can trigger calcium influx and increase intracellular calcium. Upon an increase in intracellular $\mathrm{Ca}^{2+}$, calcineurin can dephosphorylate the target nuclear factor of activated $\mathrm{T}$ cell (NFAT) protein to expose nuclear localization sequences, resulting in their rapid nuclear import (23). Moreover, $\mathrm{Ca}^{2+}$ and calcineurin-NFAT signaling has been demonstrated to be involved in regulating cell cycle (24) and apoptosis (25). In addition, Ta et al. (26) has shown that cisplatin induced up-regulation of TRPA1 mRNA both in vitro and in vivo, however, the potential molecular mechanism still required further study. Our previous study has shown that TRPA1 expressed in human renal tubular epithelial cells is upregulated during hypoxia and reoxygenation (unpublished data). Due to renal tubular cell apoptosis being the most vital mechanism of cisplatininduced acute kidney injury (AKI), we speculated that TRPA1 may mediate cisplatin-induced apoptosis in renal tubular cells via a $\mathrm{Ca}^{2+}$-dependent signaling pathway. Our study aimed to provide a new understanding of the molecular mechanism of TRPA1 in cisplatin-induced renal apoptosis.

We present the following article in accordance with the MDAR reporting checklist (available at https://dx.doi. org/10.21037/apm-21-1867).

\section{Methods}

\section{Reagents}

HC-030031 (H4415), pifithrin- $\alpha$ (P4359), and FK506 monohydrate (F4679) were all purchased from SigmaAldrich (Merck KGaA, Darmstadt, Germany). The remaining reagents used in this study were commercially available and of analytical purity.

\section{Cell culture}

HK-2 cells (CL-0109; obtained fromProcell, Wuhan, China) were cultured in keratinocyte serum-free medium (K-SFM) supplemented by $0.05 \mathrm{mg} / \mathrm{mL}$ bovine pituitary extract (Sigma, Darmstadt, Germany), $5 \mathrm{ng} / \mathrm{mL}$ human recombinant epidermal growth factor (Sigma, Darmstadt, Germany), $100 \mathrm{U} / \mathrm{mL}$ penicillin (Sigma, Darmstadt, Germany) and $100 \mathrm{~g} / \mathrm{mL}$ streptomycin (Sigma, Darmstadt, Germany). Cultures were incubated at $37{ }^{\circ} \mathrm{C}$ with $5 \%$ 
Table 1 Antibodies used and their corresponding dilution

\begin{tabular}{llcc}
\hline Reagent or resource & Source & Identifier & Dilution \\
\hline Rabbit anti-caspase-3 & Abcam & ab4051 & $1: 1,000$ \\
Rabbit anti-cleaved-cas3 & Abcam & ab2302 & $1: 1,000$ \\
Rabbit anti-p53 & Abcam & ab241566 & $1: 1,000$ \\
Rabbit anti-Bax & Abcam & ab53154 & $1: 1,000$ \\
Rabbit anti-Bcl-2 & Abcam & ab196495 & $1: 1,000$ \\
Rabbit anti-TRPA1 & Sigma & SAB1411593 & $1: 1,000$ \\
Rabbit anti-NFAT2 & Abcam & ab25916 & $1: 1,000$ \\
Rabbit anti-p-NFAT2 & Abcam & ab183023 & $1: 1,000$ \\
Rabbit anti- $\beta$-actin & Abcam & ab8227 & $1: 2,000$ \\
Goat anti-rabbit IgG H\&L & Abcam & ab6721 & $1: 5,000$ \\
\hline
\end{tabular}

TRPA1, transient receptor potential ankyrin 1; NFAT2, nuclear factor of activated T-cells 2; p-NFAT2, phospho-NFAT; IgG H\&L, immunoglobulin $\mathrm{G}$ heavy chain and light chain.

carbon dioxide $\left(\mathrm{CO}_{2}\right)(27)$.

\section{Cell viability assay}

HK-2 cells were cultured in 96 -well plates with an inoculation density of $1 \times 10^{5}$ /well for $24 \mathrm{~h}$ and then treated with DDP at concentrations of $0,5,10,20,40$, and $80 \mu \mathrm{M}$ for an additional 24, 36, or $48 \mathrm{~h}$. Subsequently, $10 \mu \mathrm{L}$ of Cell Counting Kit-8 (CCK8, Dojindo, Kumamoto, Japan) was added to the cultured cells for $2 \mathrm{~h}$, and then the absorbance was read at $450 \mathrm{~nm}$ using a microplate reader (Thermo, CA, USA).

\section{Flow cytometry}

After $48 \mathrm{~h}$ incubation with the added drugs (the TRPA1 inhibitor, HC-030031 (30 $\mu \mathrm{M})(28)$; the $\mathrm{p} 53$ inhibitor, pifithrin- $\alpha(20 \mu M)(29)$; and the calcineurin inhibitor, FK506 monohydrate $(5 \mu M)(30)$, the resuspended HK-2 cells were added with $5 \mu \mathrm{L}$ Annexin V-PE (Sigma, Darmstadt, Germany) and $5 \mu \mathrm{L}$ allophycocyanin (APC) (Sigma, Germany) for $20 \mathrm{~min}$ to incubate. The cellular apoptotic rate was tested with flow cytometry (BD FACSVerse, BD Biosciences, San Jose, CA, USA).

\section{Sodium dodecyl sulphate-polyacrylamide gel electrophoresis (SDS-PAGE) and western blot analysis}

After $48 \mathrm{~h}$ incubation with the added drugs (the TRPA1 inhibitor, HC-030031 (30 $\mu \mathrm{M})$; the p53 inhibitor, pifithrin- $\alpha(20 \mu \mathrm{M})$; and the calcineurin inhibitor, FK506 monohydrate $(5 \mu \mathrm{M})$, cell lysates were extracted with radioimmunoprecipitation assay (RIPA) buffer on treated ice-cold samples, and the protein concentrations were balanced using a bicinchoninic acid assay (BCA) protein quantification kit. SDS-PAGE and western blot analyses were performed as described by Xie et al. (31). Briefly, the balanced proteins were added with loading buffer and incubated at $100{ }^{\circ} \mathrm{C}$ for $10 \mathrm{~min}$, separated by a $10 \%$ SDS-PAGE gel and transferred to a $0.22 \mu \mathrm{m}$ polyvinylidene difluoride (PVDF) membrane. The membrane was then blocked with a mixture of trisbuffered saline and Tween 20 (TBST) containing 5\% skimmed milk (Anchor, Auckland, New Zealand) for $2 \mathrm{~h}$ at room temperature. TBST washing was applied before and after the following incubation with the primary and secondary antibodies (the antibodies and the corresponding dilution concentrations are listed in Table 1). Primary antibodies were incubated at $4{ }^{\circ} \mathrm{C}$ overnight, and the secondary antibody (goat-anti-rabbit immunoglobulin horseradish peroxidase diluted 1:5,000) was incubated for $2 \mathrm{~h}$ at room temperature. The lanes on the membrane were visualized using ECL Luminescence Kit KF001 (Affinity, Chicago, IL, USA) for $1 \mathrm{~min}$. Scan analysis was performed using a gel image analysis imaging system. Relative protein levels were calculated with the integrated optical density value (IOD) of the target protein/IOD of $\beta$-actin. 
Reverse transcriptase-polymerase chain reaction (RT-PCR)

Total ribonucleic acid (RNA) was extracted from the cell samples using the Animal Total RNA Isolation Kit RE-03014 (Foregene, Sichuan, China) according to the manufacturer's instruction. The first strand complimentary deoxyribonucleic Acid (cDNA) was synthesized using a PrimeScript RT Reagent Kit RR047A (Takara Biotechnology Co., Ltd., Dalian, China) according to the manufacturer's instruction. Real-time polymerase chain reaction (qRT- PCR) was then performed, in which the A PIKORed 96 (ThermoFisher, Waltham, MA, USA), primers, and the TB Green TM Premix Ex TaqTM II (Tli RNaseH Plus RR820A) (Takara, Kusatsu, Japan) were used (32). The relative messenger RNA (mRNA) level of each gene was presented as the fold change of target gene divided by level of $\beta$-actin, and three replicated samples were tested at the same time point. All primer sequences used in the present study were listed as follows: TRPA1, 5'-CGGCGTCCAGGTGGAGTCAATGAA-3' (Forward), 5'-TGACCTTCTGCTGCTGCGTGATGC-3' (Reverse); $\beta$-actin, 5 '-CTCCATCGTCCACCGCAAATGCTTCT-3' (Forward), 5'-CCACTGCTGTCACCTTCTCCGTTC CA-3' (Reverse).

\section{Cellular $\mathrm{Ca}^{2+}$ concentration determination}

Fluo-4/AM (2 $\mu \mathrm{M}$ in use) was used to measure the cellular $\mathrm{Ca}^{2+}$ concentration. Briefly, the HK-2 cells were cultured in 96-well plates and the indicated drugs were added for $48 \mathrm{~h}$ (the TRPA1 inhibitor, HC-030031 (30 $\mu \mathrm{M})$; the p53 inhibitor, pifithrin- $\alpha(20 \mu \mathrm{M})$; and the calcineurin inhibitor, FK506 monohydrate $(5 \mu \mathrm{M})$. The cultures were washed with Hanks Balanced Salt Solutions (HBSS) (Sigma, Darmstadt, China) three times and then processed with fluo-4/AM staining for $30 \mathrm{~min}$ at $37^{\circ} \mathrm{C}$. The cellular $\mathrm{Ca}^{2+}$ concentrations were then determined with a flow cytometer (BD FACSVerse).

\section{Statistical analysis}

All data were analyzed using one-way analysis of variance (ANOVA) or Duncan's test using the SPSS 19.0 package (SPSS Inc. Chicago, IL, USA). All quantitative results are shown as the means $\pm \mathrm{SE}$, and the differences were considered statistically non-significant, significant and extremely significant for $\mathrm{P}>0.05, \mathrm{P}<0.05$ and $\mathrm{P}<0.01$, respectively.

\section{Results}

\section{DDP reduced cell viability of $H K-2$}

To examine the HK-2 cell viability induced by DDP, HK-2 cells were added with DDP of $0,5,10,20,40$, and $80 \mu \mathrm{M}$ for 24,36 , and $48 \mathrm{~h}$. Subsequently, the CCK-8 kit was used to assess the cell viability. The results showed distinctly that $10 \mu M$ DDP is cytotoxic for HK-2 cells, and that DDP induced a decrease in cell viability in both a timedependent and dose-dependent manner (Figure 1A,1B), with $\mathrm{IC}_{50}$ values of $29.16 \mu \mathrm{M}$ at $48 \mathrm{~h}$. Using a microscope, cell morphology also showed a tendency to attenuate in a dose-dependent manner (Figure 1C).

\section{DDP induced apoptosis of HK-2 cells}

To further assess the effect of DDP on HK-2 cells, we evaluated the apoptosis of HK-2 cell treated with DDP with concentrations of $0,1 / 2 \mathrm{IC} 50$, IC50, and 2IC50 using flow cytometry. The results showed that DDP treatment sharply increased the apoptosis of HK-2 cells in a dosedependent manner after co-staining with phycoerythrin (PE) and APC (Figure 2A). To explore the mechanism underlying the apoptosis of the HK-2 cells treated with DDP, the expression of apoptosis-related proteins, such as Bax, caspase3, cleaved-cas3, p53, and Bcl-2 were measured using western blot. The results revealed that the expression of caspase3, cleaved-cas3, Bax, and p53 were enhanced after HK-2 cells were treated with DDP in a dose-dependent manner, while DDP treatment decreased the expression of Bcl-2 in a dose-dependent manner (Figure 2B).

\section{DDP promoted the expression of TRPA1}

Our previous study showed that hypoxia and reoxygenation significantly increases the expression of TRPA1. To further evaluate the underlying function of TRPA1 on AKI, we detected TRPA1 expression both at the mRNA and protein levels using qRT-PCR and western blot, respectively. Likewise, the results show that DDP induced TRPA1 expression in a dose-dependent manner at both the mRNA and protein levels (Figure 3A,3B).

\section{DDP increased cytosolic $\mathrm{Ca}^{2+}$ in $\mathrm{HK}-2$ cells}

As a non-selective calcium-permeable channel, the activation of TRPA1 can trigger calcium influx and increase intracellular calcium. Given DDP could induce 
A

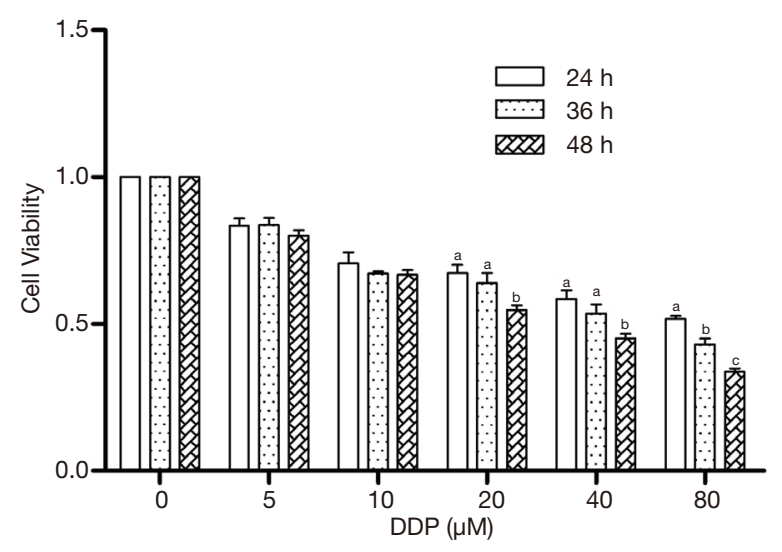

B

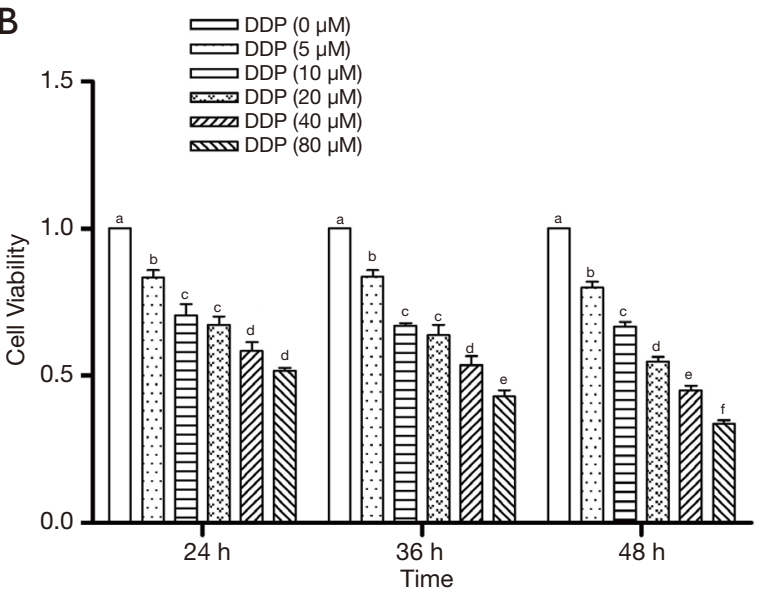

C

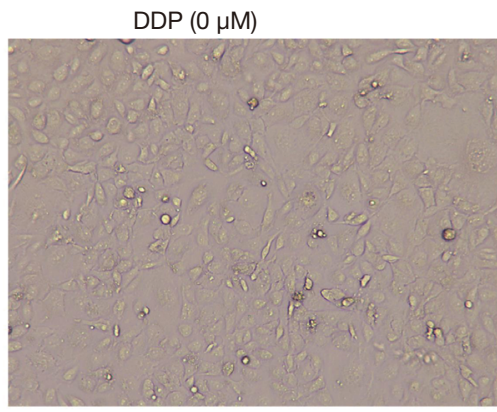

$\operatorname{DDP}(20 \mu \mathrm{M})$

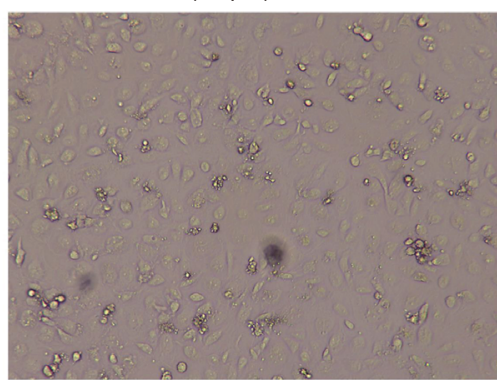

$\operatorname{DDP}(5 \mu \mathrm{M})$

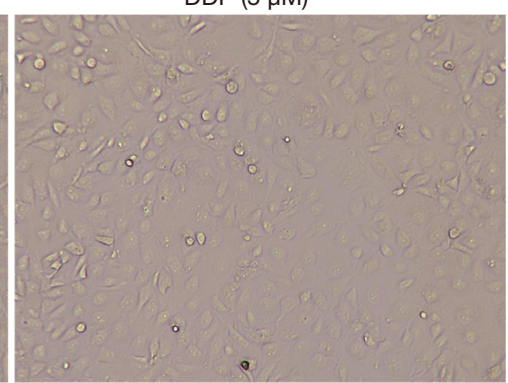

$\operatorname{DDP}(40 \mu \mathrm{M})$

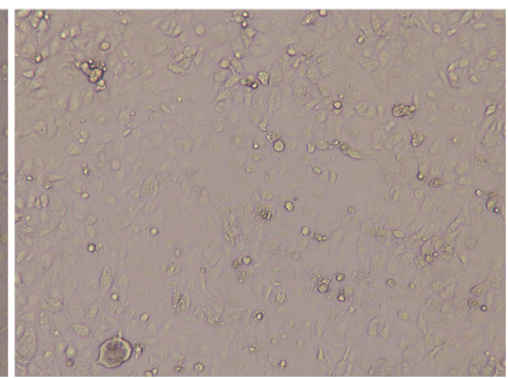

$\operatorname{DDP}(10 \mu \mathrm{M})$

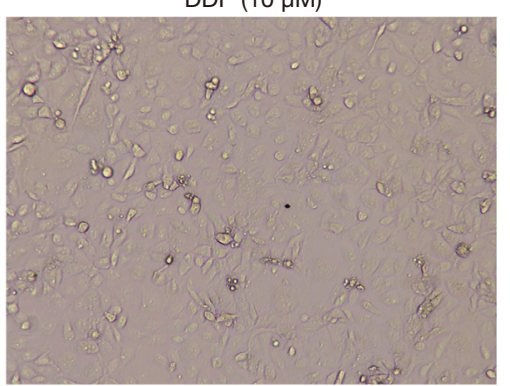

$\operatorname{DDP}(80 \mu \mathrm{M})$

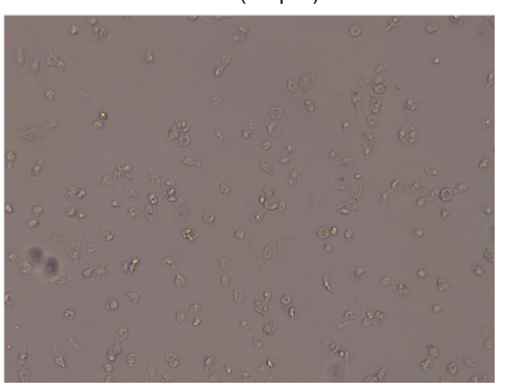

Figure 1 DDP inhibits the HK-2 cell viability. HK-2 cells were added with DDP of concentrations of $0,5,10,20,40$, and $80 \mu M$ for 24 , 36 and $48 \mathrm{~h}$. DDP decreased the cell viability in a time-dependent (A) and dose-dependent manner (B). Cell morphology was directly observed at $48 \mathrm{~h}$ using an inverted light microscope, magnification, $\times 100(\mathrm{C})$. The mean \pm SEM of three independent samples for each group is shown. The difference between groups is statistically significant $(\mathrm{P}<0.05)$ where the letters over the bars are different, as determined by ANOVA. Different letters (a, b, c, d, e and f) between groups indicate that the differences between groups are significant. DDP, diamminedichloroplatinum; SEM, standard error of mean; ANOVA, one-way analysis of variance.

the expression of TRPA1, Fluo-4/AM staining and a flow cytometer was used to detect intracellular calcium concentration in HK-2 cells after DDP treatment. Similarly, the results confirmed that DDP treatment augmented intracellular calcium concentration in a dose-dependent manner (Figure 4A,4B).

\section{HC-030031 relieved HK-2 cells apoptosis}

As a powerful TRPA1 antagonist, HC-030031 was used 

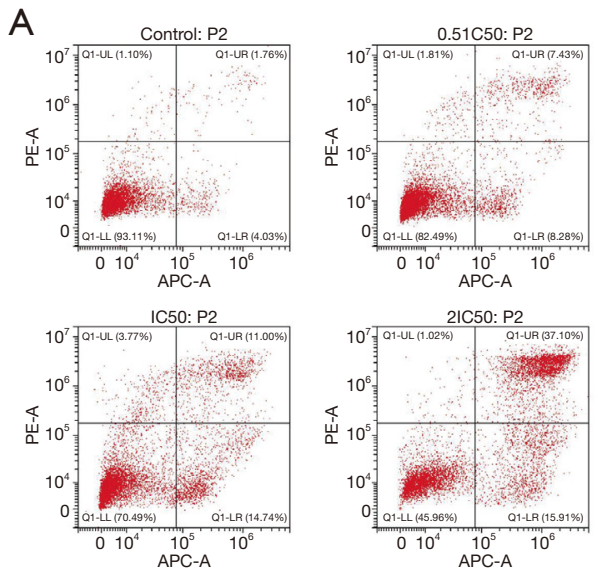

B

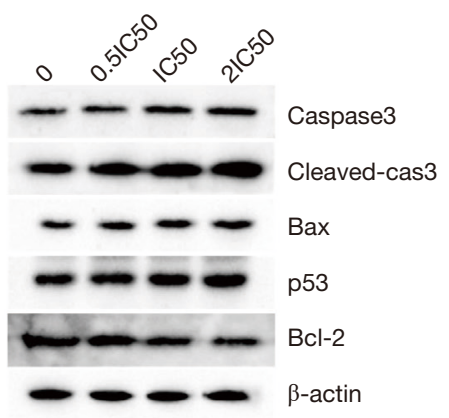

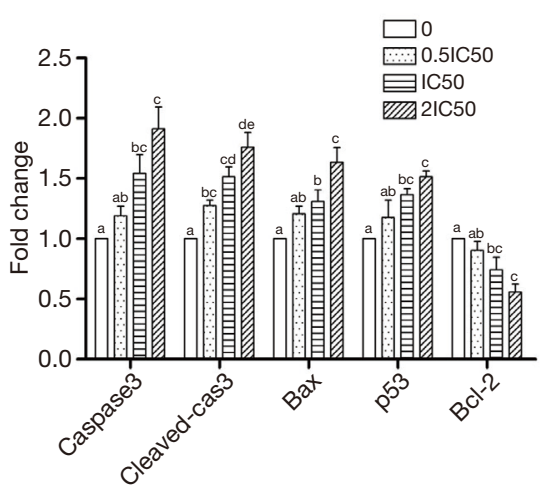

Figure 2 DDP induced apoptosis and the expression of apoptosis-related proteins. HK-2 cells were treated with DDP with the concentrations of 0, 0.5IC50 (14.58 $\mu \mathrm{M})$, IC50 $(29.16 \mu \mathrm{M})$, and 2IC50 (58.32 $\mu \mathrm{M})$ (all IC50 mentioned in this study refer to the IC50 of the HK-2 cells treated with DDP for 48 h). The apoptotic rate of the HK-2 cells was detected using flow cytometry after PE and APC costaining (A), and the expression of caspase-3, cleaved-cas3, Bax, p53, and Bcl-2 protein were determined using western blot (B). The mean \pm SEM of three independent samples for each group was shown. The difference between groups is statistically significant $(\mathrm{P}<0.05)$ where the letters (a, b, c, d and e) over the bars are different, as determined by ANOVA. DDP, diamminedichloroplatinum; SEM, standard error of mean; ANOVA, one-way analysis of variance; PE, phycoerythrin; APC, allophycocyanin.
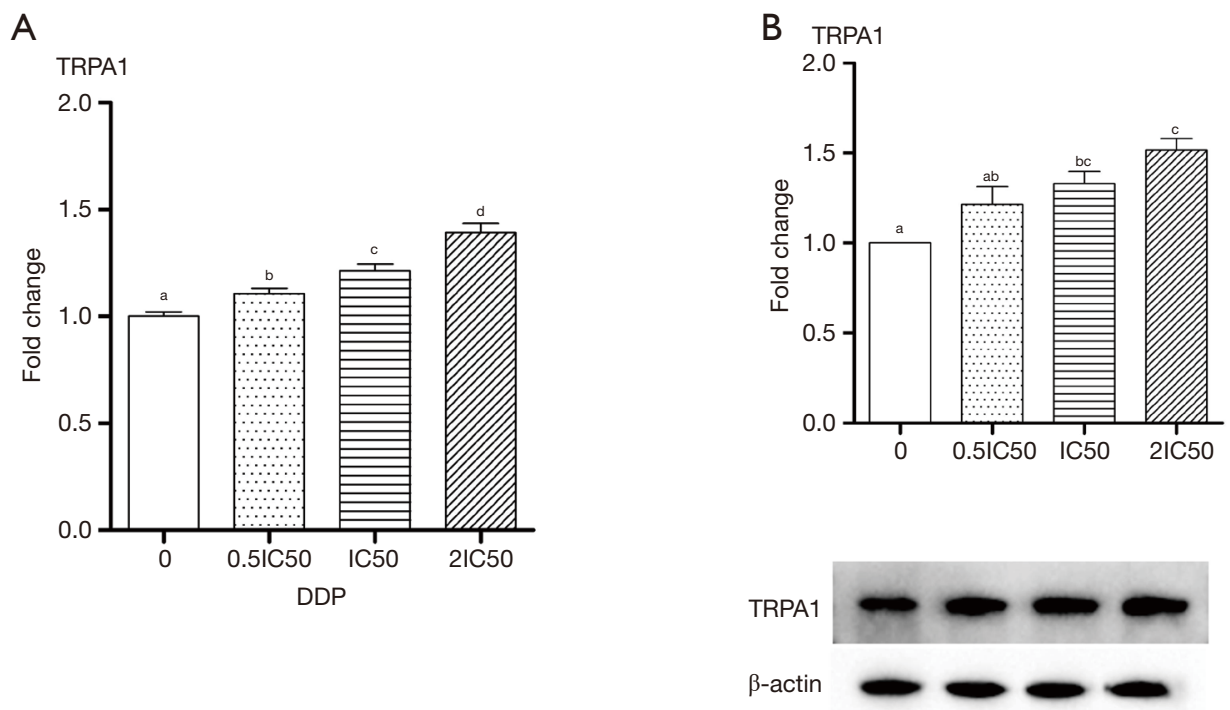

Figure 3 TRPA1 expression was enhanced in a dose-dependent manner at both the mRNA and protein levels. HK-2 cells were treated with DDP with concentrations of 0, 0.5IC50 (14.58 $\mu \mathrm{M})$, IC50 (29.16 $\mu \mathrm{M})$, and 2IC50 (58.32 $\mu \mathrm{M})$. The expression of TRPA1 was detected using qRT-PCR (A), and TRPA1 expression was examined using western blot analysis (B). The mean \pm SEM of three independent samples for each group are shown. The differences between groups are statistically significant $(\mathrm{P}<0.05)$ where the letters $(\mathrm{a}, \mathrm{b}$, c, d) over the bars are different, as determined by ANOVA. TRPA1, transient receptor potential ankyrin 1; DDP, diamminedichloroplatinum; SEM, standard error of mean; ANOVA, one-way analysis of variance; mRNA, messenger ribonucleic acid; qRT-PCR, real-time polymerase chain reaction. 

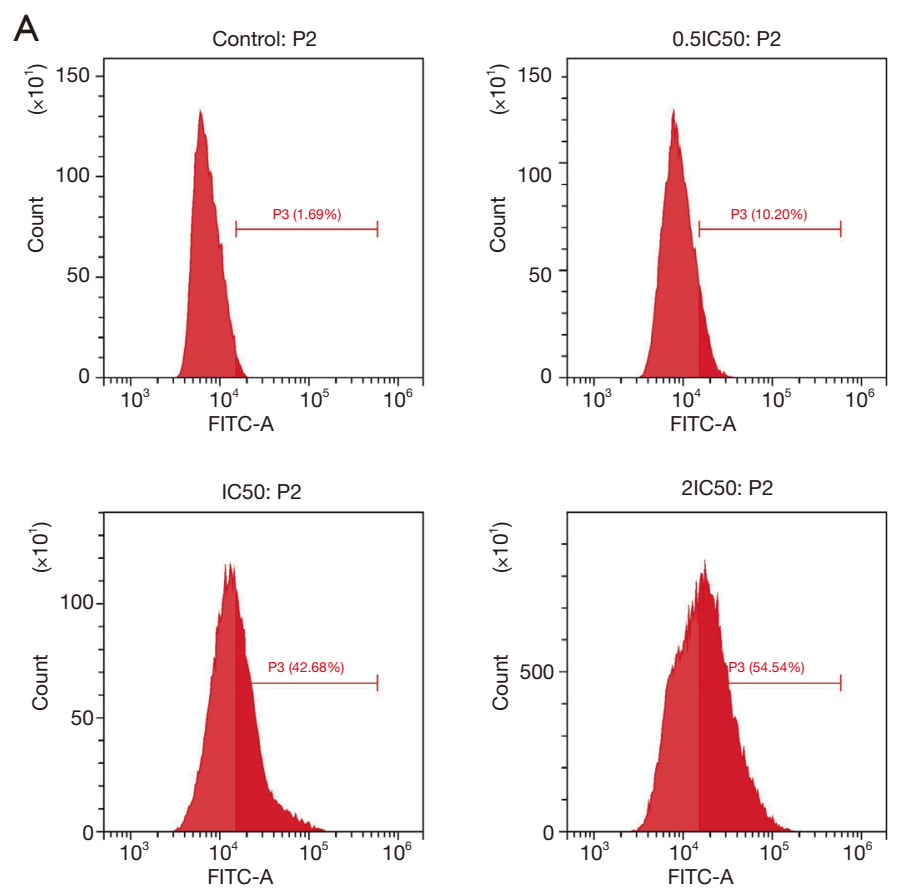

B

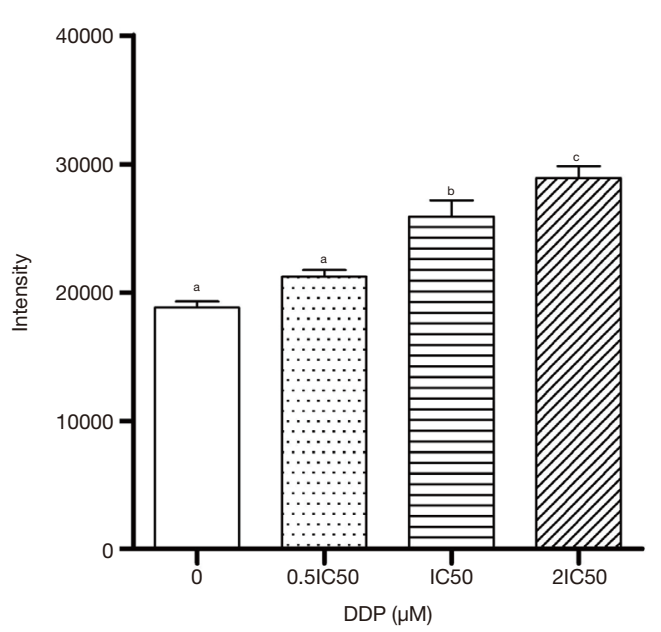

Figure 4 DDP treatment boosted the intracellular calcium concentration in a dose-dependent manner. HK-2 cells were treated with DDP with concentrations of 0 , 0.5 IC50 $(14.58 \mu \mathrm{M})$, IC50 $(29.16 \mu \mathrm{M})$, and 2IC50 (58.32 $\mu \mathrm{M})$. (A) The intracellular calcium concentration was detected using fluo-4/AM with flow cytometer. (B) The relative intensity of the calcium concentration was shown as a bar graph. The mean \pm SEM of three independent samples for each group are shown. The difference between groups is statistically significant $(\mathrm{P}<0.05)$ where the letters $(\mathrm{a}, \mathrm{b}, \mathrm{c})$ over the bars are different, as determined by ANOVA. TRPA1, transient receptor potential ankyrin 1; DDP, diamminedichloroplatinum; SEM, standard error of mean; ANOVA, one-way analysis of variance.

to examine the effect on apoptosis and the expression of apoptosis-related proteins. HC-030031 treatment alleviated the HK-2 cell apoptosis caused by DDP, and the use of pifithrin- $\alpha$, which functioned as a $\mathrm{p} 53$ inhibitor, also relieved HK-2 cell apoptosis induced by DDP (Figure $5 A$ ). Interestingly, simultaneous treatment of HK-2 cells with HC-030031 and pifithrin- $\alpha$ led to a further reduction on the basis of mitigation caused by HC-030031 or pifithrin- $\alpha$ induced by DDP (Figure $5 A$ ). The expression of apoptosisrelated proteins (Bax, caspase3, cleaved-cas3, p53, and Bcl2) was also determined using western blot. Uniformly, HC030031 or pifithrin- $\alpha$ decreased the expression of Bax, caspase3, cleaved-cas3, and $\mathrm{p} 53$, while they increased that of Bcl-2 induced by DDP (Figure 5B). HC-030031 and pifithrin- $\alpha$ used together further reduced the expression of Bax, caspase3, cleaved-cas3, and p53, while they enhanced that of $\mathrm{Bcl}-2$ induced by DDP (Figure $5 B$ ). More importantly, the use of HC-030031 and/or pifithrin- $\alpha$ all reduced the expression of TRPA1 in DDP-induced
HK-2 cells (Figure 5B). Therefore, the results suggest that TRPA1-mediated cisplatin-induced apoptosis in renal tubular cells via 533 signaling pathway.

\section{HC-030031 reduced the cytosolic $\mathrm{Ca}^{2+}$}

The concentration of cytosolic $\mathrm{Ca}^{2+}$ was evaluated with the TRPA1 antagonist HC-030031 using fluo-4/AM with flow cytometer. The intracellular calcium concentration was significantly lower than that after HC-030031 was used, while it was lower than that with pifithrin- $\alpha$ in the DDP-induced HK-2 cells. Consistently, the intracellular calcium concentration was significantly lower than that for simultaneous treatment with HC-030031 and pifithrin- $\alpha$ in HK-2 cells induced by DDP (Figure 6A,6B).

\section{FK506 alleviated HK-2 cell apoptosis}

As a serine/threonine protein phosphatase regulated by 
A

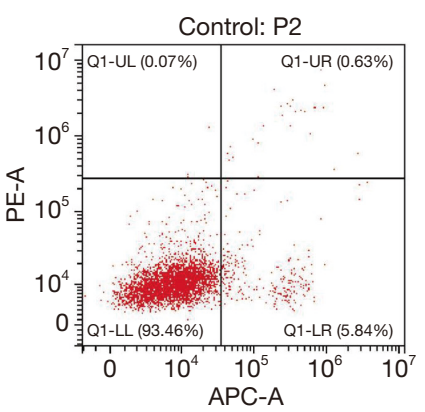

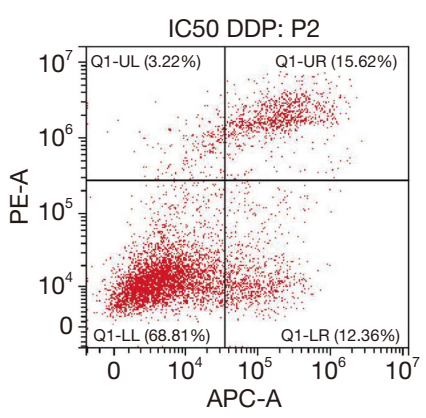
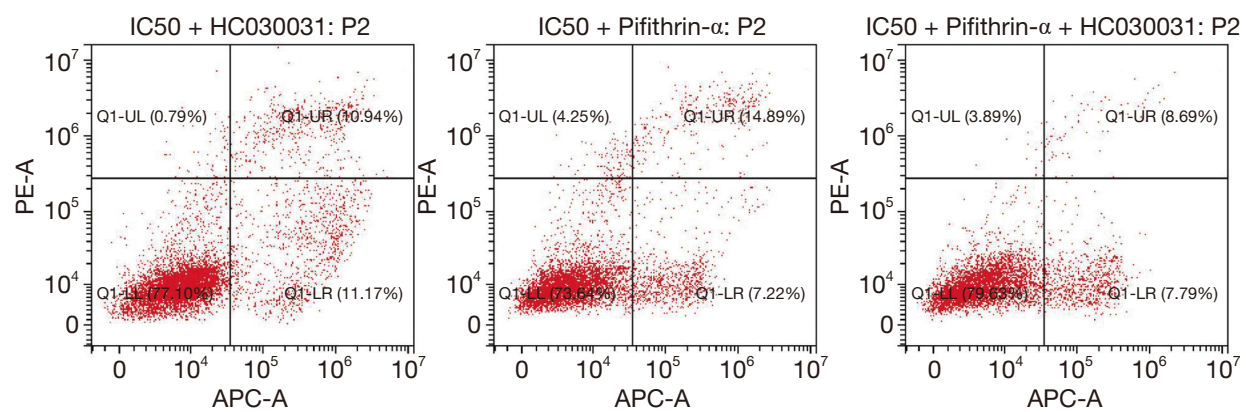

B

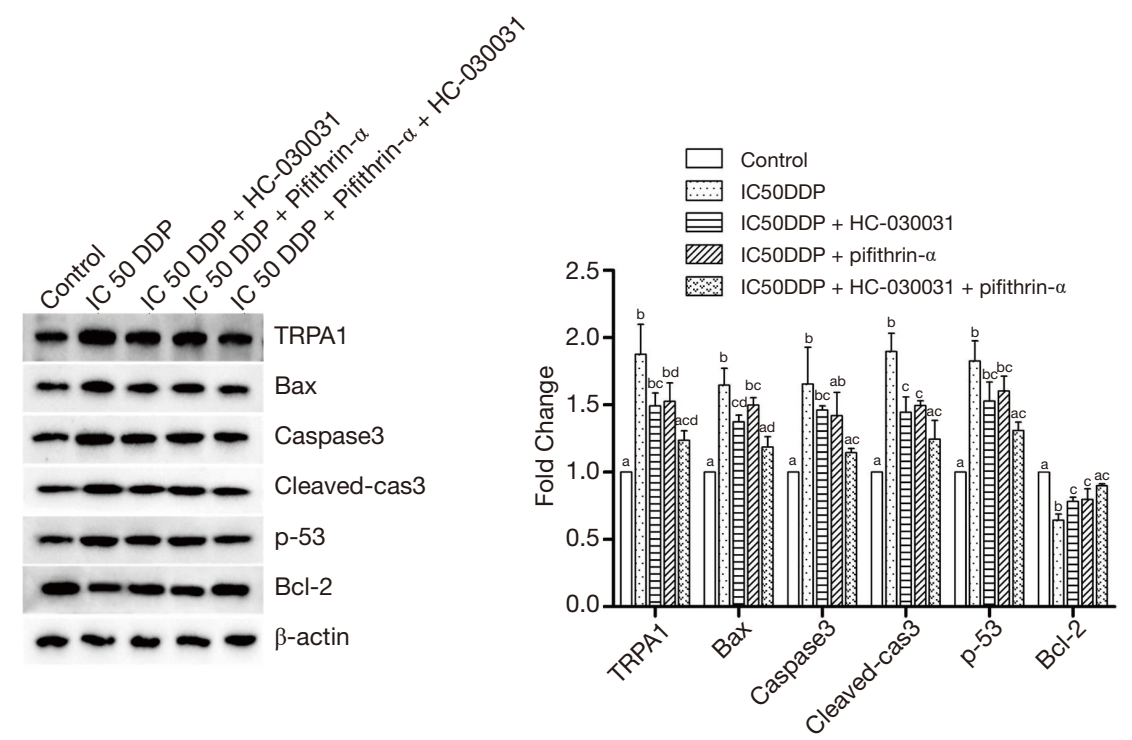

Figure 5 The use of HC-030031 and/or pifithrin- $\alpha$ alleviated HK-2 cell apoptosis and the expression of apoptosis-related proteins caused by DDP. HK-2 cells were treated with IC50 DDP, IC50 DDP + HC-03003, IC50 DDP + pifithrin- $\alpha$, IC50 DDP + pifithrin- $\alpha$ + HC-03003 or PBS (control) (IC50 DDP $=29.16 \mu \mathrm{M}$ DDP). The apoptosis of the HK-2 cells was detected using flow cytometry after PE and APC co-staining (A), and the expression of TRPA1, Bax, caspase-3, cleaved-cas3, p53, and Bcl-2 was determined using western blot (B). The mean \pm SEM of three independent samples for each group are shown. The difference between groups is statistically significant $(\mathrm{P}<0.05)$ where the letters (a, b, c, d) over the bars are different, as determined by ANOVA. TRPA1, transient receptor potential ankyrin 1; DDP, diamminedichloroplatinum; PBS, phosphate buffered saline; SEM, standard error of mean; ANOVA, one-way analysis of variance; PE, phycoerythrin; APC, allophycocyanin. 
A

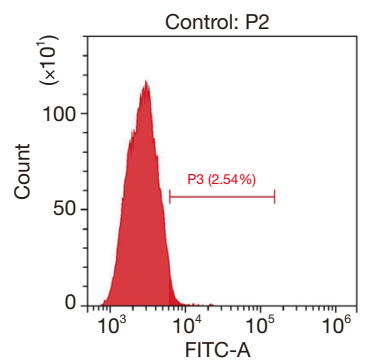

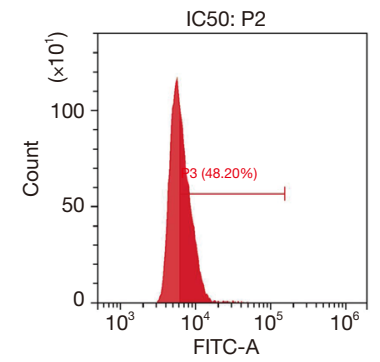

B

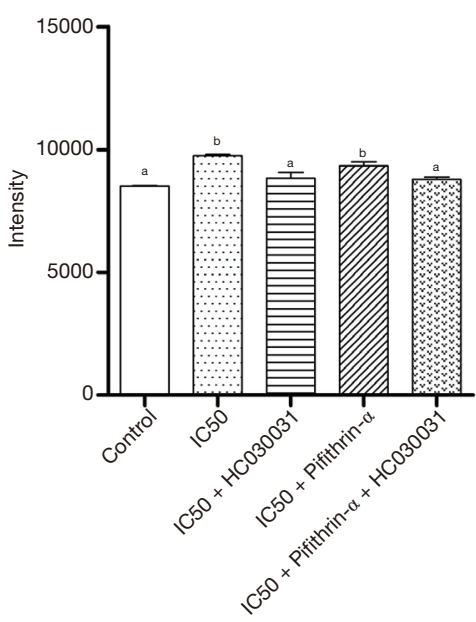

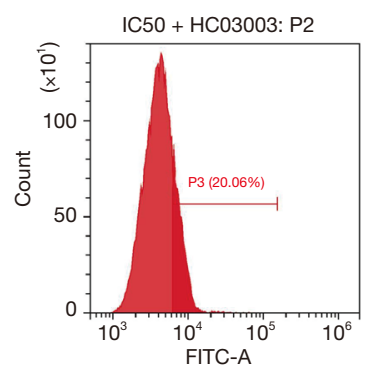
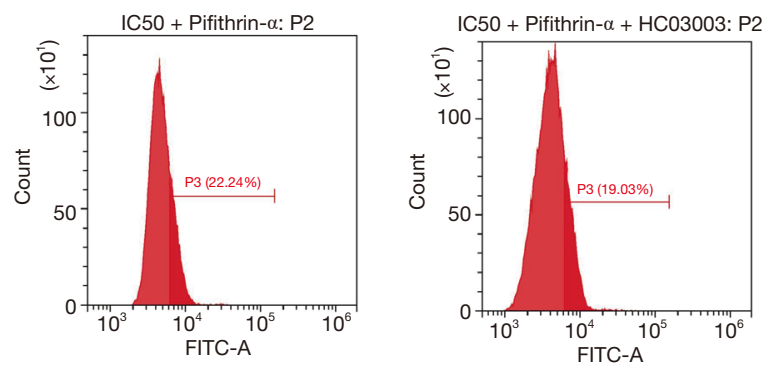

Figure 6 The use of HC-030031 and/or pifithrin- $\alpha$ decreased the intracellular calcium concentration induced by DDP. HK-2 cells were treated with IC50 DDP, IC50 DDP + HC-03003, IC50 DDP + pifithrin- $\alpha$, IC50 DDP + pifithrin- $\alpha+$ HC-03003 or PBS (control) (IC50 $\mathrm{DDP}=29.16 \mu \mathrm{M}$ DDP). (A) The intracellular calcium concentration was detected used fluo-4/AM with flow cytometer. (B) The relative intensity of the calcium concentration was shown as a bar graph. The mean \pm SEM of three independent samples for each group are shown. The difference between groups is statistically significant $(\mathrm{P}<0.05)$ where the letters $(\mathrm{a}, \mathrm{b})$ over the bars are different, as determined by ANOVA. DDP, diamminedichloroplatinum; PBS, phosphate buffered saline; SEM, standard error of mean; ANOVA, one-way analysis of variance.

calcium and calmodulin $(\mathrm{CaM})$, calcineurin is a canonical transponder related to cell cycle progression and cell apoptosis. We assessed the effect of calcineurin on apoptosis using the calcineurin inhibitor FK506. Treatment with HC-030031 reduced HK-2 cell apoptosis caused by DDP, and calcineurin inhibitor FK506 also alleviated HK-2 cell apoptosis induced by DDP. Compared with HC-030031 or FK506 group, the apoptosis of HK-2 cell in HC-030031 combined with FK506 group was significantly decreased (Figure 7A). Consistently, HC-030031 and FK506 decreased the expression of Bax, caspase3, cleaved-cas3, and $\mathrm{p} 53$, while they increased that of $\mathrm{Bcl}-2$ induced by DDP (Figure 5B). HC-030031 and FK506 used simultaneously further reduced the expression of Bax, caspase3, cleavedcas3, and $\mathrm{p} 53$, while they enhanced that of $\mathrm{Bcl}-2$ induced by DDP (Figure $7 B$ ). Hence, the results suggested that TRPA1-mediated cisplatin-induced apoptosis might be related to the calcineurin signal pathway.

\section{HC-030031 and FK506 declined the expression of NFAT}

Since the calcineurin can dephosphorylate the target protein NFAT after activation by $\mathrm{Ca}^{2+}$, we further discuss the effect of HC-030031 and FK506 on NFAT protein. The results showed that HC-030031, pifithrin- $\alpha$, or FK506 decreased the expression of NFAT and phosphorylation of NFAT, while the use of HC-030031 combined with pifithrin- $\alpha$ or FK506 further reduced the expression of NFAT and phosphorylation of NFAT (Figure 8A,8B). Thus, the results suggest that TRPA1mediated cisplatin-induced apoptosis might be related to the calcineurin/NFAT signal pathway.

\section{Discussion}

Cisplatin is most successful drug in chemotherapy treatment for many malignant solid cancers. However, its side-effects are also non-negligible for clinicians; especially its nephrotoxicity remains a major obstacle that limits the use and efficacy of cisplatin in cancer therapy. In the present study, we found that DDP could induce apoptosis, TRPA1 expression, and intracellular calcium concentrations of HK-2 cells. Pharmacological block with HC-030031, pifithrin- $\alpha$, or FK506 could counteract apoptosis, 
A
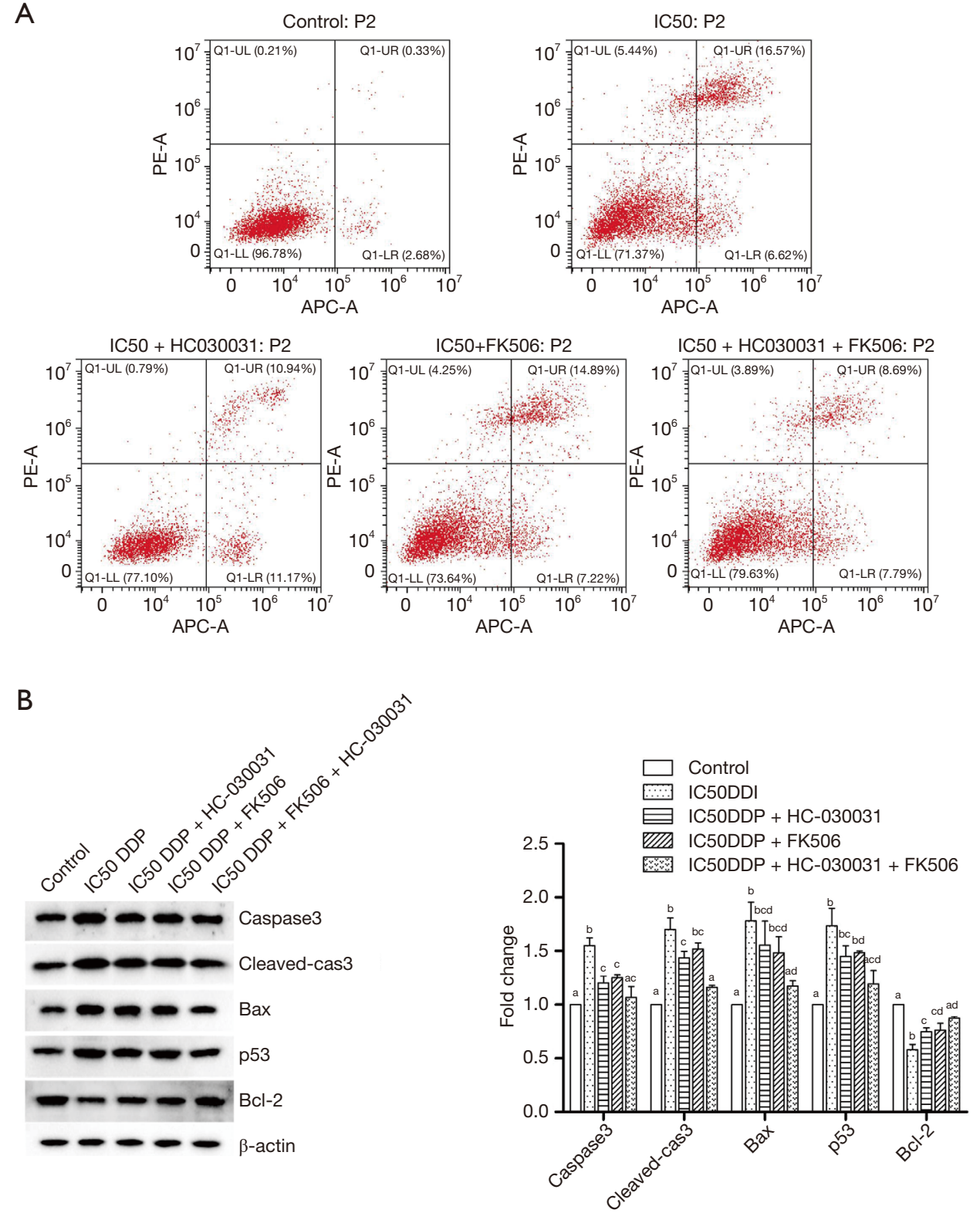

Figure 7 The use of HC-030031 and/or FK506 alleviated HK-2 cell apoptosis and the expression of apoptosis-related proteins caused by DDP. HK-2 cells were treated with IC50 DDP, IC50 DDP + HC-03003, IC50 DDP + FK506, IC50 DDP + FK506 + HC-03003, or PBS (control) (IC50 DDP =29.16 $\mu \mathrm{M}$ DDP). The apoptosis of HK-2 cells was detected using flow cytometry after simultaneous staining with PE and APC (A), and the expression of caspase-3, cleaved-cas3, Bax, p53, and Bcl-2 was determined using western blot (B). The mean \pm SEM of three independent samples for each group are shown. The difference between groups is statistically significant $(\mathrm{P}<0.05)$ where the letters (a, b, c, d) over the bars are different, as determined by ANOVA. DDP, diamminedichloroplatinum; PBS, phosphate buffered saline; SEM, standard error of mean; ANOVA, one-way analysis of variance; PE, phycoerythrin; APC, allophycocyanin. 
A

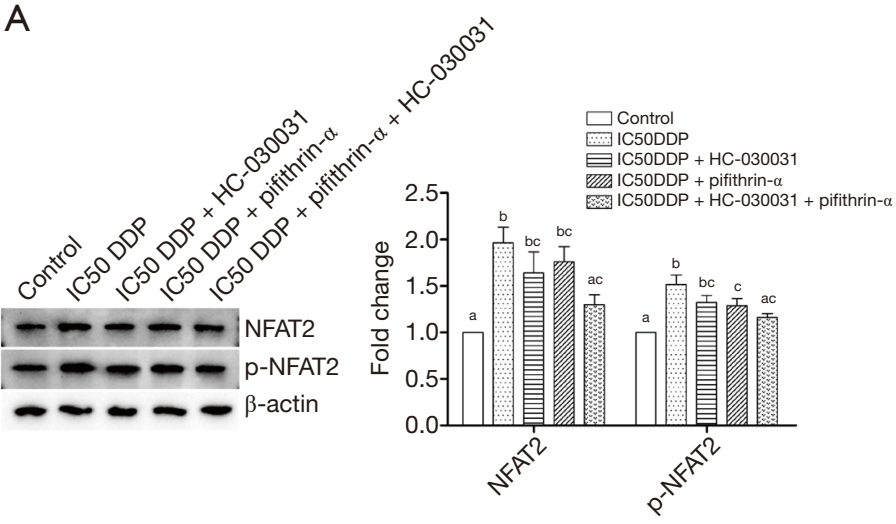

B

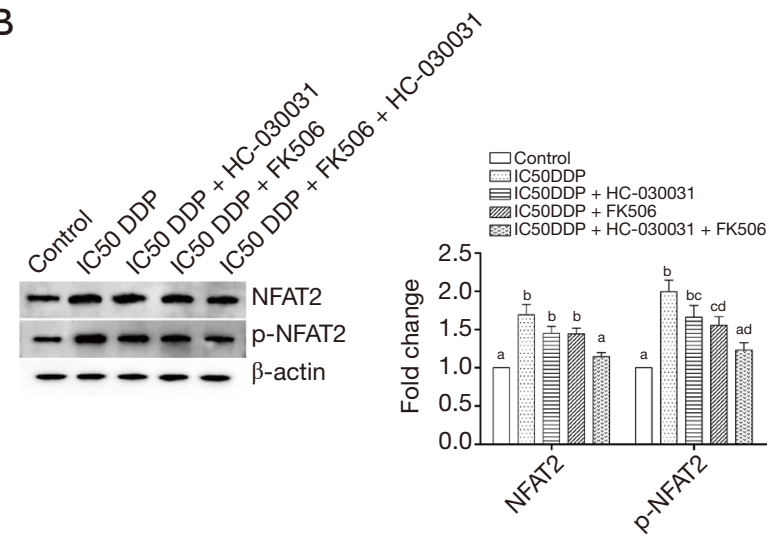

Figure 8 The use of HC-030031 combined with pifithrin- $\alpha$ or FK506 decreased the expression of NFAT caused by DDP. HK-2 cells were treated with IC50 DDP, IC50 DDP + HC-03003, IC50 DDP + pifithrin- $\alpha$, IC50 DDP + pifithrin- $\alpha$ + HC-03003 or PBS (control) (A), and IC50 DDP, IC50 DDP + HC-03003, IC50 DDP + FK506, IC50 DDP + FK506 + HC-03003, or PBS (control) (IC50 DDP =29.16 $\mu M$ DDP) (B). The expression of NFAT and p-NFAT was determined using western blot. The mean \pm SEM of three independent samples for each group are shown. The difference between groups is statistically significant $(\mathrm{P}<0.05)$ where the letters $(\mathrm{a}, \mathrm{b}, \mathrm{c}, \mathrm{d})$ over the bars are different, as determined by ANOVA. TRPA1, transient receptor potential ankyrin 1; NFAT2, nuclear factor of activated T-cells 2; p-NFAT2, phosphoNFAT; DDP, diamminedichloroplatinum; PBS, phosphate buffered saline; SEM, standard error of mean; ANOVA, one-way analysis of variance.

intracellular calcium, and the expression of NFAT and p-NFAT of HK-2 cells induced by DDP. The use of HC030031 combined with pifithrin- $\alpha$ or FK506 further alleviated the above-mentioned effects. Taken together, our research demonstrated that TRPA1 may mediate cisplatininduced apoptosis in renal tubular cells via the calcineurinNFAT2-p53 signaling pathway.

Cisplatin has many potential mechanisms of renal injury, including oxidative stress (33), inflammatory response (34), apoptosis (35), DNA damage (36), and others $(6,37)$. Among them, apoptosis is the most important mechanism that causes nephrotoxicity. Cisplatin-induced nephrotoxicity involves two major apoptotic pathways. One is an endogenous pathway including the endoplasmic reticulum, mitochondria, and other organelles (35), and the other is an exogenous pathway. The endogenous pathway has become a vital factor in tubular cell death. During the process of cisplatin-induced apoptosis, the expression of pro-apoptotic proteins in the Bcl-2 family increases and the expression of anti-apoptotic proteins decreases (35). Thus, it has been shown that cisplatin induced the apoptosis of HK-2 cell accompanied with the increase of Bax, caspase3, cleaved-cas3, and p53, and the decrease of Bcl-2 in a dosedependent manner.

TRPA1 is a non-selective calcium-permeable channel involved in the calcium-regulated signaling pathways (38). Our previous study revealed that the hypoxia and reoxygenation that can imitate AKI significantly increase the expression of TRPA1, which suggests that TRPA1 might play an important role in AKI. Therefore, we assessed the effect on the TRPA1 expression of HK-2 cells induced by cisplatin. The results verified that cisplatin induced the expression of TRPA1 in a dose-dependent manner. The activation of TRPA1 can trigger calcium influx and increase intracellular calcium. Since cisplatin can induce the expression of TRPA1, we speculated that the intracellular calcium concentration after treatment with cisplatin should also increase in a dose-dependent manner. The use of fluo-4/AM with flow cytometer verified this conjecture. Moreover, the pharmacological inhibition of TRPA1 with HC-030031 (39) can alleviate apoptosis, the expression of TRPA1, and intracellular calcium concentration induced by cisplatin. Together, TRPA1 might mediate cisplatin-induced apoptosis in renal tubular cells via the $\mathrm{Ca}^{2+}$-dependent signaling pathway.

$\mathrm{Ca}^{2+}$ is an important second messenger that plays an important role in cell cycle progression (24) and cell apoptosis (23). When the cisplatin-induced TRPA1 expression, calcium influx, and intracellular calcium increase, some calcium-regulated signaling pathways may 
be activated. Calcineurin is a serine/threonine protein phosphatase regulated by calcium, and calmodulin functions as a major transponder of the $\mathrm{Ca}^{2+}$-dependent signal transduction cascade (40). Due to the inhibition of the phosphatase activity of calcineurin by FK506 (41), pharmacological blocking of calmodulin through FK506 further reduces apoptosis on the basis of HC-030031 inhibits apoptosis, suggesting that TRPA1-mediated cisplatin-induced apoptosis might be related to the calcineurin signal pathway.

Calcineurin activated by calcium transmits signals to the nucleus through the dephosphorylation and translocation of NFAT transcription factors (23). Both HC-030031 and FK506 can inhibit the expression of NFAT, which suggests that TRPA1-mediated cisplatin-induced apoptosis might be related to the calcineurin/NFAT signal pathway. Furthermore, P53 inhibitor pifithrin- $\alpha$ (42) can also have the same effect on apoptosis and protein expression. Briefly, TRPA1-mediated cisplatin-induced apoptosis might be related to the calcineurin/NFAT/p53 signal pathway.

In summary, the present study demonstrated that cisplatin can induce apoptosis of HK-2 cells, which might be related to TRPA1 through the calcineurin/NFAT/p53 signal pathway. However, there were also some limitations in the present study. For example, overexpression or knockdown of TRPA1 should be performed to further verify the functions of TRPA1. We hope our research can provide novel insights into the molecular mechanism of TRPA1 in cisplatin-induced apoptosis.

\section{Acknowledgments}

Funding: This work was supported by the Sichuan Science and Technology Program (2019YFH0069), Sichuan Medical Research Project (S20014), and Chengdu Medical Research Project (2020208).

\section{Footnote}

Reporting Checklist: The authors have completed the MDAR reporting checklist. Available at https://dx.doi. org/10.21037/apm-21-1867

Data Sharing Statement: Available at https://dx.doi. org/10.21037/apm-21-1867

Conflicts of Interest: All authors have completed the ICMJE uniform disclosure form (available at https:// dx.doi.org/10.21037/apm-21-1867). All authors report funding from the Sichuan Science and Technology Program (2019YFH0069), Sichuan Medical Research Project (S20014), and Chengdu Medical Research Project (2020208). The authors have no other conflicts of interest to declare.

Ethical Statement: The authors are accountable for all aspects of the work in ensuring that questions related to the accuracy or integrity of any part of the work are appropriately investigated and resolved.

Open Access Statement: This is an Open Access article distributed in accordance with the Creative Commons Attribution-NonCommercial-NoDerivs 4.0 International License (CC BY-NC-ND 4.0), which permits the noncommercial replication and distribution of the article with the strict proviso that no changes or edits are made and the original work is properly cited (including links to both the formal publication through the relevant DOI and the license). See: https://creativecommons.org/licenses/by-nc-nd/4.0/.

\section{References}

1. Rosenberg B, Vancamp L, Krigas T. Inhibition of Cell Division in Escherichia Coli by Electrolysis Products from a Platinum Electrode. Nature 1965;205:698-9.

2. Wang D, Lippard SJ. Cellular processing of platinum anticancer drugs. Nat Rev Drug Discov 2005;4:307-20.

3. Cohen SM, Lippard SJ. Cisplatin: from DNA damage to cancer chemotherapy. Prog Nucleic Acid Res Mol Biol 2001;67:93-130.

4. Arany I, Safirstein RL. Cisplatin nephrotoxicity. Semin Nephrol 2003;23:460-4.

5. Siddik ZH. Cisplatin: mode of cytotoxic action and molecular basis of resistance. Oncogene 2003;22:7265-79.

6. Pabla N, Dong Z. Cisplatin nephrotoxicity: mechanisms and renoprotective strategies. Kidney Int 2008;73:994-1007.

7. Hartmann JT, Fels LM, Knop S, et al. A Randomized Trial Comparing the Nephrotoxicity of Cisplatin/IfosfamideBased Combination Chemotherapy with or without Amifostine in Patients with Solid Tumors. Invest New Drugs 2000;18:281-9.

8. Hartmann JT, Lipp HP. Toxicity of platinum compounds. Expert Opin Pharmacother 2003;4:889-901.

9. Sastry J, Kellie SJ. Severe neurotoxicity, ototoxicity and nephrotoxicity following high-dose cisplatin and 
amifostine. Pediatr Hematol Oncol 2005;22:441-5.

10. Pasetto LM, D'Andrea MR, Brandes AA, et al. The development of platinum compounds and their possible combination. Crit Rev Oncol Hematol 2006;60:59-75.

11. Cornelison TL, Reed E. Nephrotoxicity and hydration management for cisplatin, carboplatin, and ormaplatin. Gynecol Oncol 1993;50:147-58.

12. Bajorin DF, Bosl GJ, Alcock NW, et al. Pharmacokinetics of cis-diamminedichloroplatinum(II) after administration in hypertonic saline. Cancer Res 1986;46:5969-72.

13. Beyer J, Rick O, Weinknecht S, et al. Nephrotoxicity after high-dose carboplatin, etoposide and ifosfamide in germcell tumors: incidence and implications for hematologic recovery and clinical outcome. Bone Marrow Transplant 1997;20:813-9.

14. Miller RP, Tadagavadi RK, Ramesh G, et al. Mechanisms of Cisplatin nephrotoxicity. Toxins (Basel) 2010;2:2490-518.

15. Song KI, Park JY, Lee S, et al. Protective effect of tetrahydrocurcumin against cisplatin-induced renal damage: in vitro and in vivo studies. Planta Med 2015;81:286-91.

16. Faubel S, Lewis EC, Reznikov L, et al. Cisplatininduced acute renal failure is associated with an increase in the cytokines interleukin (IL)-1beta, IL-18, IL-6, and neutrophil infiltration in the kidney. J Pharmacol Exp Ther 2007;322:8-15.

17. Lieberthal W, Triaca V, Levine J. Mechanisms of death induced by cisplatin in proximal tubular epithelial cells: apoptosis vs. necrosis. Am J Physiol 1996;270:F700-8.

18. Lee RH, Song JM, Park MY, et al. Cisplatin-induced apoptosis by translocation of endogenous Bax in mouse collecting duct cells. Biochem Pharmacol 2001;62:1013-23.

19. Nilius B, Prenen J, Owsianik G. Irritating channels: the case of TRPA1. J Physiol 2011;589:1543-9.

20. Zygmunt PM, Hogestatt ED. Trpa1. Handb Exp Pharmacol 2014;222:583-630.

21. Koivisto A, Chapman H, Jalava N, et al. TRPA1: a transducer and amplifier of pain and inflammation. Basic Clin Pharmacol Toxicol 2014;114:50-5.

22. Zhu M, Chen L, Zhao P, et al. Store-operated $\mathrm{Ca}(2+)$ entry regulates glioma cell migration and invasion via modulation of Pyk2 phosphorylation. J Exp Clin Cancer Res 2014;33:98.

23. Dewenter M, von der Lieth A, Katus HA, et al. Calcium Signaling and Transcriptional Regulation in Cardiomyocytes. Circ Res 2017;121:1000-20.
24. Fric J, Lim CX, Mertes A, et al. Calcium and calcineurin-NFAT signaling regulate granulocytemonocyte progenitor cell cycle via Flt3-L. Stem Cells 2014;32:3232-44.

25. Jayanthi S, Deng X, Ladenheim B, et al. Calcineurin/ NFAT-induced up-regulation of the Fas ligand/ Fas death pathway is involved in methamphetamineinduced neuronal apoptosis. Proc Natl Acad Sci U S A 2005;102:868-73.

26. Ta LE, Bieber AJ, Carlton SM, et al. Transient Receptor Potential Vanilloid 1 is essential for cisplatin-induced heat hyperalgesia in mice. Mol Pain 2010;6:15.

27. Azad MA, Roberts KD, Yu HH, et al. Significant accumulation of polymyxin in single renal tubular cells: a medicinal chemistry and triple correlative microscopy approach. Anal Chem 2015;87:1590-5.

28. Muraki K, Sekine T, Ando Y, et al. An environmental pollutant, 9,10-phenanthrenequinone, activates human TRPA1 via critical cysteines 621 and 665. Pharmacol Res Perspect 2017;5:e00342.

29. Yang A, Liu F, Guan B, et al. p53 induces miR-199a-3p to suppress mechanistic target of rapamycin activation in cisplatin-induced acute kidney injury. J Cell Biochem 2019;120:17625-34.

30. Bennett J, Cassidy H, Slattery C, et al. Tacrolimus Modulates TGF- $\beta$ Signaling to Induce EpithelialMesenchymal Transition in Human Renal Proximal Tubule Epithelial Cells. J Clin Med 2016;5:50.

31. Xie Y, Chen S, Yan Y, et al. Potential of recombinant inorganic pyrophosphatase antigen as a new vaccine candidate against Baylisascaris schroederi in mice. Veterinary Research 2013;44:90.

32. Wang T, Huang W, Costa MM, et al. Two copies of the genes encoding the subunits of putative interleukin (IL)4/IL-13 receptors, IL-4R $\alpha$, IL-13R $\alpha 1$ and IL-13R $\alpha 2$, have been identified in rainbow trout (Oncorhynchus mykiss) and have complex patterns of expression and modulation. Immunogenetics 2011;63:235-53.

33. Kuhad A, Tirkey N, Pilkhwal S, et al. Renoprotective effect of Spirulina fusiformis on cisplatin-induced oxidative stress and renal dysfunction in rats. Ren Fail 2006;28:247-54.

34. Ramesh G, Reeves WB. TNF-alpha mediates chemokine and cytokine expression and renal injury in cisplatin nephrotoxicity. J Clin Invest 2002;110:835-42.

35. Park MS, De Leon M, Devarajan P. Cisplatin induces apoptosis in LLC-PK1 cells via activation of mitochondrial pathways. J Am Soc Nephrol 2002;13:858-65. 
36. dos Santos NA, Carvalho Rodrigues MA, Martins NM, et al. Cisplatin-induced nephrotoxicity and targets of nephroprotection: an update. Arch Toxicol 2012;86:1233-50.

37. Cullen KJ, Yang Z, Schumaker L, et al. Mitochondria as a critical target of the chemotheraputic agent cisplatin in head and neck cancer. J Bioenerg Biomembr 2007;39:43-50.

38. Bley K, Boorman G, Mohammad B, et al. A comprehensive review of the carcinogenic and anticarcinogenic potential of capsaicin. Toxicol Pathol 2012;40:847-73.

39. Eid SR, Crown ED, Moore EL, et al. HC-030031, a TRPA1 selective antagonist, attenuates inflammatory- and neuropathy-induced mechanical hypersensitivity. Mol Pain
2008;4:48.

40. Clipstone NA, Crabtree GR. Identification of calcineurin as a key signalling enzyme in T-lymphocyte activation. Nature 1992;357:695-7.

41. Im SH, Rao A. Activation and deactivation of gene expression by $\mathrm{Ca} 2+$ /calcineurin-NFAT-mediated signaling. Mol Cells 2004;18:1-9.

42. Lin Y, Waldman BC, Waldman AS. Suppression of high-fidelity double-strand break repair in mammalian chromosomes by pifithrin-alpha, a chemical inhibitor of p53. DNA Repair (Amst) 2003;2:1-11.

(English Language Editor: B. Meiser)
Cite this article as: Meng X, Zhang Y, Ma Y, Du Y, Gan C, Feng J, Deng F. Transient receptor potential ankyrin 1 mediates cisplatin-induced apoptosis in renal tubular cells via calciumdependent signaling pathway. Ann Palliat Med 2021;10(8):90259038. doi: 10.21037/apm-21-1867 